\title{
Breast cancer and pyoderma gangrenosum: a complication after conservative surgery and radiotherapy
}

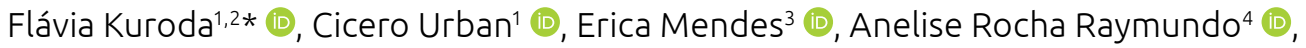 \\ Alessandra Amatuzzi Cordeiro Fornazari' (1), Teodora Roballo Durigan 5 (D)
}

\section{ABSTRACT}

Pyoderma gangrenosum (PG) is a rare, ulcerative, and painful neutrophilic dermatosis of unknown cause associated with systemic diseases and/or pathergy phenomenon in $30 \%$ of cases. We report the case of a breast cancer patient submitted to oncoplastic conservative surgery followed by adjuvant radiotherapy, with long-term progression to PG. It's rare and challeng ing nature reinforces the need for early diagnosis to increase treatment effectiveness and reduce morbidity.

KEYWORDS: Pyoderma gangrenosum. Breast cancer. Radiotherapy. Breast conserving surgery. Corticoids.

\section{INTRODUCTION}

Pyoderma gangrenosum $(\mathrm{PG})$ is a dermatological inflammatory disease resulting from innate immune system dysfunction, with highly heterogeneous presentation and course ${ }^{1,2}$. It is a rare neutrophilic dermatosis characterized by papule, pustule, and vesicle formation rapidly progressing to painful skin ulcers, often located in the lower limbs, although they have been reported on the head, breast, oral cavity, trunk, perineum, and upper limbs ${ }^{1,3}$. These skin lesions present well-defined edges, peripheral erythema, moist base, subcutaneous tissue necrosis, painful high sensitivity, suppuration, and occasional bleeding ${ }^{4,5}$. The disease presents great morbidity, and its course may be chronic or recurrent.

Although they may occur spontaneously, more than $50 \%$ of lesions develop due to skin hyperactivity at trauma sites, with special emphasis on postoperative ones (PPG ${ }^{6.7}$. Multiple case reports have described the progress of $\mathrm{PG}$ after cosmetic, oncologic, and reconstructive breast surgery, but few $\mathrm{PG}$ reports address breast cancer after conservative surgery associated with radiotherapy.

\section{CASE REPORT}

This case report describes a 50-year-old Caucasian, nulligravida patient with a history of hiatus hernia, dyslipidemia, and hypothyroidism, taking omeprazole, simvastatin, and levothyroxine. She also had a previous history of fibroids hysterectomy surgery, and a family history of breast cancer (her mother died at the age of 50 years).

The patient had a T2NOM0 left breast cancer - grade 2 invasive ductal subtype, triple-negative, and Ki-67 40\%. She received neoadjuvant chemotherapy (CT) (doxorubicin and cyclophosphamide, followed by taxane - AC-T + carboplatin), which ended on February 6, 2018. On March 19, 2018, she underwent quadrantectomy + sentinel lymph node biopsy (SLNB) on the left side and bilateral oncoplastic surgery, using the lower pedicle technique (Figure 1). On the $15^{\text {th }}$ postoperative day, the patient developed small dehiscence in the left breast $\mathrm{T}$ area, which was resutured. The wound healed completely, and the patient was referred to radiotherapy. She received left-breast external conformational radiotherapy at a total dose of $50 \mathrm{~Gy}$ (30 fractions) and a $60 \mathrm{~Gy}$ boost (30 fractions), ending on July 11, 2018. The patient progressed well with grade 1 radiodermatitis in the treated area.

In October $2019\left(19^{\text {th }}\right.$ postoperative month and $15^{\text {th }}$ post-radiotherapy month), she developed small periareolar ulceration on the left breast (Figure 2). At that time, infection was suspected, and the patient was treated with debridement, Hydrofiber dressing with silver and non-adherent membrane, and antibiotic therapy

'Mastology Department, Hospital Nossa Senhora das Graças - Curitiba (PR), Brazil.

2Post-graduation Program in Biotechnology, Universidade Positivo - Curitiba (PR), Brazil.

${ }^{3}$ CLINIPAM - Curitiba (PR), Brazil.

${ }^{4}$ Dermatology Department, Santa Casa de Misericórdia de Curitiba - Curitiba (PR), Brazil.

${ }^{5}$ Universidade Positivo - Curitiba (PR), Brazil.

*Corresponding author: flaviakuroda@hotmail.com

Conflict of interests: nothing to declare.

Received on: 06/16/2020. Accepted on: 07/16/2020 


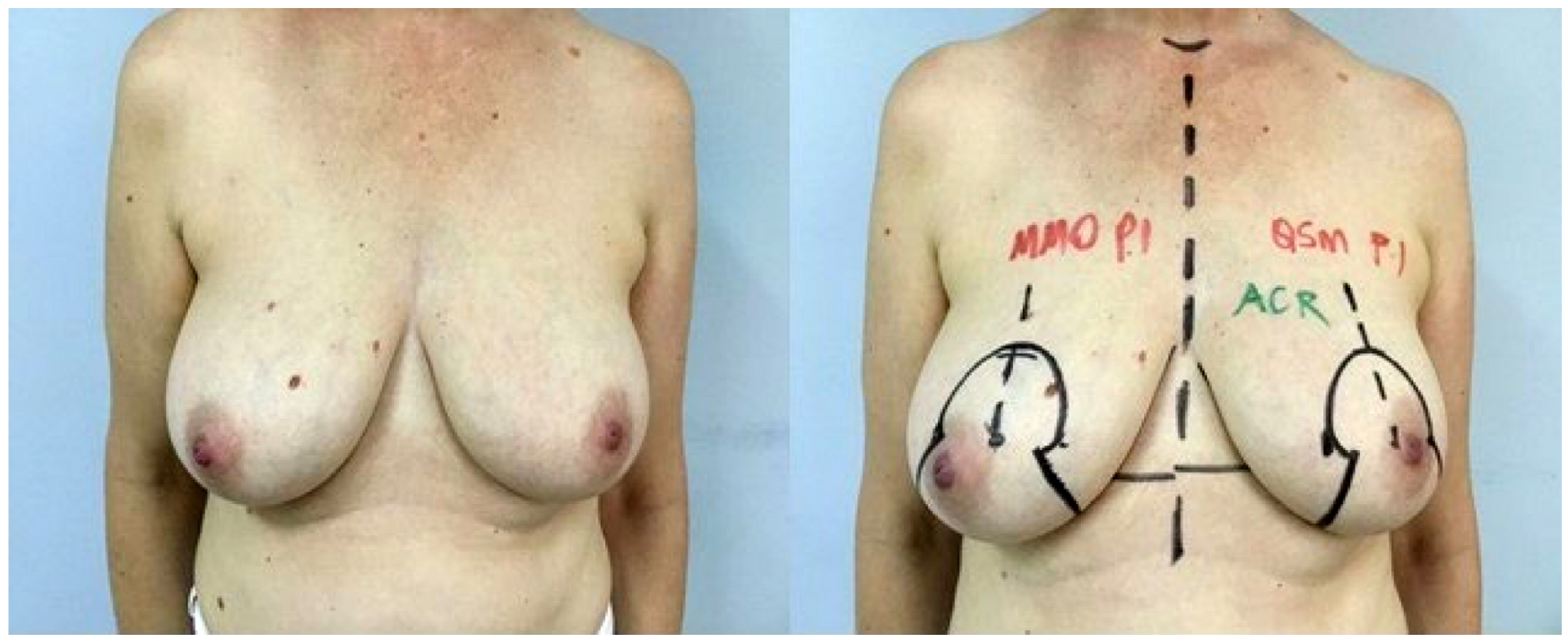

Figure 1. Preoperative surgical planning.

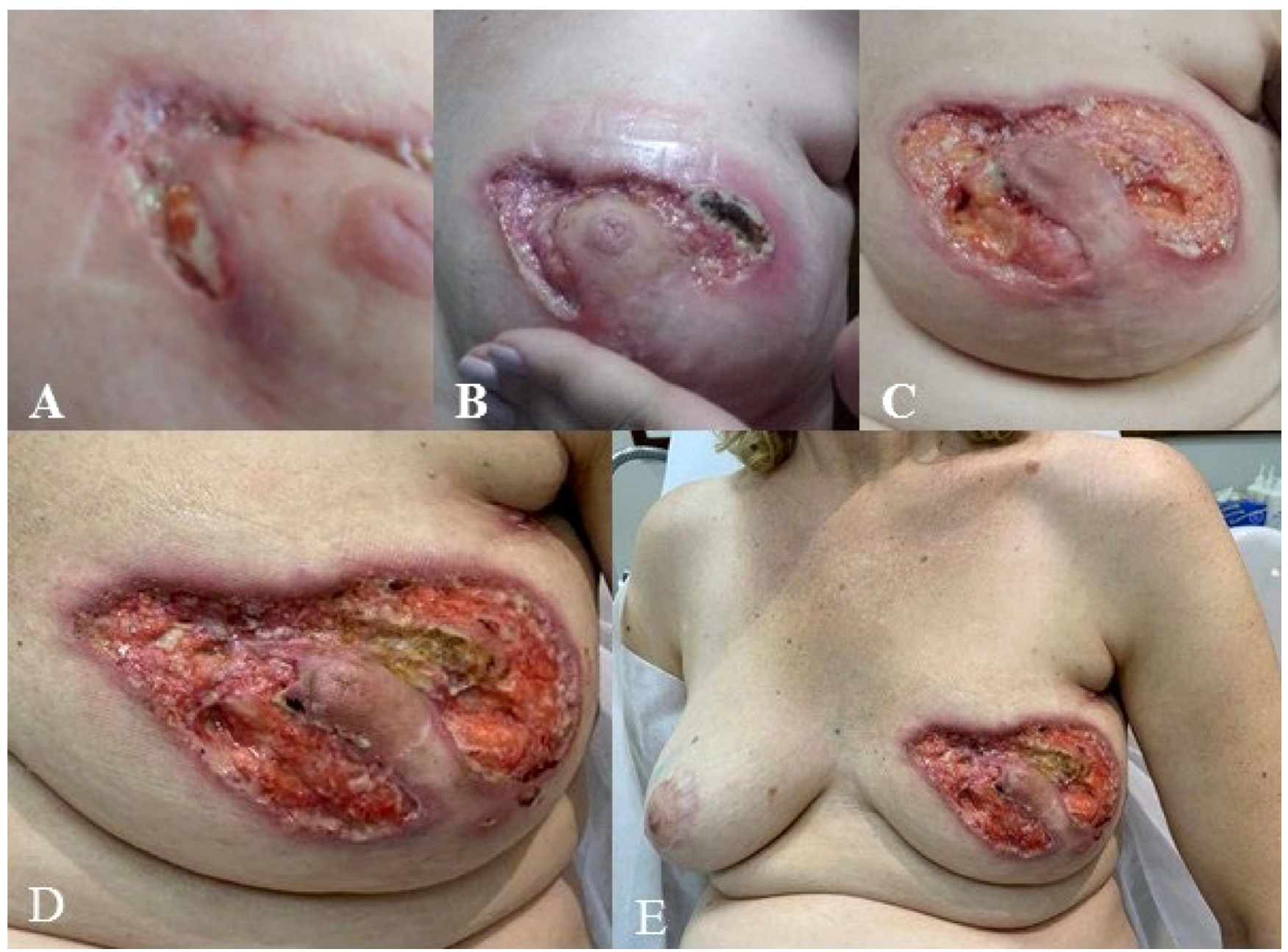

Figure 2. Pyoderma gangrenosum lesion progression. (A and B) October 2019 (19th postoperative month and $15^{\text {th }}$ post-radiotherapy month). (C) November 2019: ulcer progression with necrosis foci. (D and E) December 2019: ulcer involving the entire breast, excluding the nipple and part of the areola. 
(cefadroxil) for 21 days. The crusted ulcer gradually progressed, with necrotic foci and intense pain (Figure 2). In December 2019, the lesion had affected the entire breast, excluding the nipple and part of the areola (Figure 2). The patient was taking dipyrone, naproxen, and codeine/paracetamol, without pain control, and receiving wound dressing care.

On December 4, 2019, she was admitted for complementary tests, culture collection, and incisional biopsy. On that occasion, laboratory tests, upper endoscopy, colonoscopy, bone scintigraphy, and chest, abdominal, and pelvic computed tomography were performed, all of them without evidence of abnormalities. Based on the clinical history and progress, $\mathrm{PG}$ was the main diagnostic hypothesis, and an empirical treatment was started with oral prednisone at $80 \mathrm{mg}$ once a day + local use of a porous regeneration membrane during hospitalization. On the $15^{\text {th }}$ day of corticotherapy, the patient reported $70 \%$ to $80 \%$ pain improvement.

Histopathological results showed moderate epithelial hyperplasia, as well as chronic and severe acute neutrophilic inflammation. General bacterioscopy and mycobacteria and fungi culture were negative, but common germ culture was positive for Burkholderia cepacia and Citrobacter freundii complex.

During oral corticosteroid treatment, tiredness, weight gain, and lower limb pain were the patient's main complaints. One month after treatment, she reported significant pain reduction and progressive improvement in wound appearance. In a period of two months using corticosteroid associated with Protopic $^{\circledast}$ (tacrolimus), the wound had small residual ulcerated areas at the lesion edges (Figure 3). In three months, she was completely healed (Figure 3). Oral corticosteroid weaning was then initiated, firstly with $60 \mathrm{mg}$ for 14 days, followed by $40 \mathrm{mg}$ for another 14 days, and finally, $20 \mathrm{mg}$ for 14 days. The patient completed corticosteroid weaning in May 2020, and her wound is now completely healed (Figure 3).

\section{DISCUSSION}

PG is considered a rare disease, with an estimated prevalence of 3 cases per 100,000 people, and 0.63 new cases diagnosed per year per 100,000 people1. The disease presents a slight female predominance, and its incidence peak occurs between 20 and 50 years of age, with children and adolescents representing only $4 \%$ of cases $^{3}$. PG pathogenesis is not well known, but the condition is associated with underlying diseases, such as inflammatory bowel disease, rheumatoid arthritis, psoriatic arthritis, autoimmune hepatitis, hidradenitis suppurativa, acne, and hematologic disorders, in $50 \%$ to $70 \%$ of cases $^{8,9}$. In the present context, the patient had no previous history of these underlying diseases, and nothing significant was identified during the investigation.

PG diagnosis is mainly clinical and can be exclusionary, especially in case of a previous wound history, subjecting the patient to repeated antibiotic therapy and ineffective debridements ${ }^{10-12}$. PG is currently classified into four clinical subtypes, based on its morphology: classic (ulcerative), bullous, pustular, and vegetative ${ }^{1}$. These subtypes may coexist, but in general, the classical form is the most common, with pain being one of the main symptoms in this case ${ }^{7}$. Although they may occur spontaneously, more than $50 \%$ of lesions develop due to skin hyperactivity at trauma sites, with special emphasis on PPG, i.e., in these cases (30\%), the pathergy phenomenon is essential ${ }^{6,7}$. In PPG, after a period of typical appearance (between four and six weeks), the
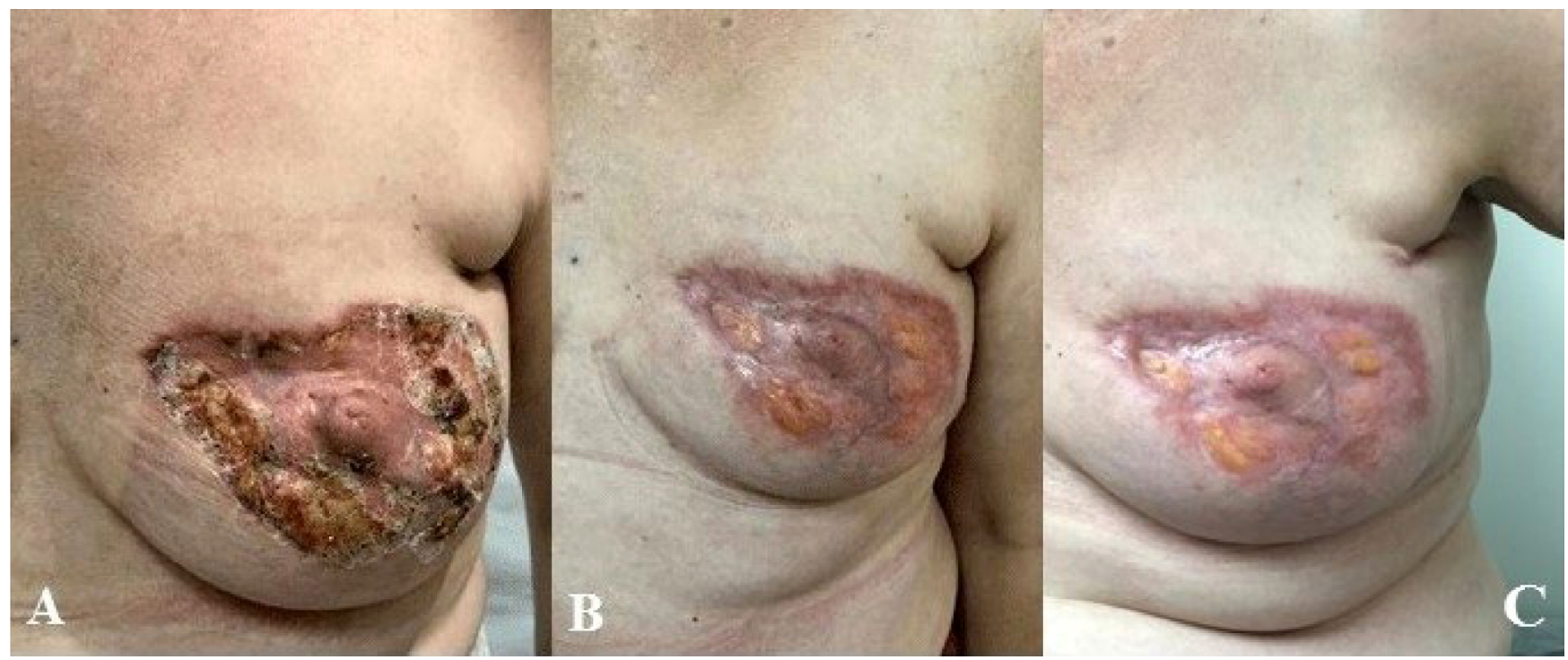

Figure 3. Pyoderma gangrenosum lesion progression after the start of corticotherapy. (A) 2 months of treatment: small ulcerated areas at the lesion edges. (B) 3 months of treatment: healed wound and start of corticosteroid weaning. (C) Complete corticosteroid post-weaning: fully healed wound. 
surgical wound shows small dehiscence that usually coalesce into large ulceration areas in a process that goes beyond the surgical wound. Granulation tissue is practically non-existent, and pain is inconstant.

In general, breasts are an unusual site for PG manifestation, but we underline that approximately $80 \%$ of known breast PG cases are postoperative ones ${ }^{13,14}$. In a systematic review that included 87 PPG cases followed by cosmetic and reconstructive breast surgery, most of them (44\%) occurred after reduction surgery, and $16 \%$ after breast reconstruction by microsurgery $y^{15}$. A total of 32 cases (37\%) were associated with breast cancer and $17 \%$ with autoimmune diseases ${ }^{15}$. In another review based on Latin American statistics from 1981 to 2018, 96 out of 232 PG cases were found in Brazill. Only 11 of these cases were associated with breast procedures (eight breast reductions, one breast implant, one phyllodes tumor, and one postquadrantectomy case) $)^{1}$. The case described above presented a classical morphological progression (ulcerative), starting at the periareolar incision and extending throughout the breast, excluding the nipple. Contrary to the specialized literature, the lesion developed later, after the pathergy phenomenon 19 months after cancer surgery.

PG has no gold standard treatment due to a lack of randomized controlled studies; however, the method most frequently reported is based exclusively on systemic steroid administration, followed by the combination of systemic steroids and corticosteroid-sparing agents ${ }^{3,16}$. Possible options include dexamethasone, cyclosporine, colchicine, thalidomide, sulfonamide, azathioprine, mycophenolate mofetil, tumor necrosis factor $\alpha$ (TNF- $\alpha$ ) inhibitors, calcineurin inhibitors, immunoglobulin, and surgery ${ }^{3}$.
In a systematic review on post-breast surgery PG, the most common treatments were steroids with 73 cases (84\%) and/or cyclosporine $\mathrm{A}(22 \%)^{15}$. A few cases employed infliximab $(\mathrm{n}=2)$, tacrolimus $(\mathrm{n}=3)$, adalimumab $(\mathrm{n}=1)$, and hyperbaric oxygen therapy $(\mathrm{n}=4)$. Rapid response to immunosuppressive therapy was reported in most cases, with a mean treatment duration of 4.7 months. Skin grafting was performed in 19 patients, and local rotation or free flap in $11^{15}$. The case described showed a rapid response to steroid and complete lesion remission after three months of treatment, even though the breast had been previously irradiated.

\section{CONCLUSION}

PG is rare and challenging for the differential diagnosis of breast diseases. Knowledge related to clinical presentation, predisposing factors, and risk surgical conditions can contribute to early diagnosis and avoiding progress to extremely severe as well as treatment-resistant cases.

\section{AUTHORS' CONTRIBUTIONS}

F.K.: study concept, data curation, formal analysis, methodology, project management, writing - review \& editing.

C.U.: study concept, data curation, formal analysis, methodology, project management, writing - review \& editing.

E.M.: data curation, methodology.

A.R.R: data curation, methodology.

A.A.C.F.: research, validation, formal analysis, writing - review \& editing.

T.R.D.: research, writing - original draft.

\section{REFERENCES}

1. Rodríguez-Zúñiga MJM, Heath MS, Gontijo JRV, OrtegaLoayza AG. Pyoderma gangrenosum: a review with special emphasis on Latin America literature. An Bras Dermatol. 2019;94(6):729-43. http://dx.doi.org/10.1016/j. abd.2019.06.001

2. Mella JR, Maselli AM, Guo L. A Deceptive Diagnosis: Pyoderma Gangrenosum After Breast Surgery-A Case Series and Literature Review. Ann Plast Surg. 2019;83(Supl. 4):S21-30. http://dx.doi.org/10.1097/SAP.0000000000002101

3. Kechichian E, Haber R, Mourad N, El Khoury R, Jabbour S, Tomb R. Pediatric pyoderma gangrenosum: a systematic review and update. Int J Dermatol. 2017;56(5):486-95. http:// dx.doi.org/10.1111/ijd.13584

4. Maverakis E, Ma C, Shinkai K, Fiorentino D, Callen JP, Wollina U, et al. Diagnostic criteria of ulcerative Pyoderma Gangrenosum - A Delphi Consensus of international experts. JAMA Dermatol. 2018;154(4):461-6. http://dx.doi.org/10.1001/ jamadermatol.2017.5980
5. BrunstingLA, GoeckermanWH,O'LearyPA.Pyoderma(ecthyma) gangraenosum - clinical and experimental observations in five cases occurring in adults. Arch Derm Syphilol. 1930;22(4):655-80. http://dx.doi.org/10.1001/archderm.1930.01440160053009

6. Billings SD. Common and critical inflammatory dermatoses every pathologist should know. Mod Pathol. 2020;33:107-17. http://dx.doi.org/10.1038/s41379-019-0400-z

7. Bonamigo RR, Razera F, Olm GS. Dermatoses neutrofílicas - Parte I. An Bras Dermatol. 2011;86(1):11-27. https://doi. org/10.1590/S0365-05962011000100002

8. Alavi A, French LE, Davis MD, Brassard A, Kirsner RS. Pyoderma Gangrenosum: An Update on Pathophysiology, Diagnosis and Treatment. Am J Clin Dermatol. 2017;18(3):35572. http://dx.doi.org/10.1007/s40257-017-0251-7

9. Kandula P, Shah K, Wolverton JE, Le C, Wolverton ES. Pyoderma gangrenosum: a presenting sign of myelodysplastic syndrome in undiagnosed Fanconi anemia. Dermatol Online J. 2019;25(1):13030/qt9xj8b544. 
10. Guaitoli G, Piacentini F, Omarini C, Andreotti A, Palma E, Papi $\mathrm{S}$, et al. Post-surgical pyoderma gangrenosum of the breast: needs for early diagnosis and right therapy. Breast Cancer. 2019;26(4):520-3. http://dx.doi.org/10.1007/s12282-018-00940-5

11. Gosch MC, Guaya IP, Medina MR, Stefanazzi M, Pinilla JP, Leyton GM, et al. Pioderma Gangrenoso de la Mama: Reporte de un caso y revisión de la literatura. Rev Chil Dermatol. 2012;28(4):439-43.

12. Weenig RH, Davis MDP, Dahl PR, Su WPD. Skin ulcers misdiagnosed as pyoderma gangrenosum. N Engl J Med. 2002;347(18):1412-8. http://dx.doi.org/10.1056/NEJMoa013383

13. Tomoda Y, Kagawa S, Kurata S, Tanaka K. Pyoderma gangrenosum of the breast. BMJ Case Rep. 2018;11(1):e228243. http://dx.doi.org/10.1136/bcr-2018-228243
14. Tuffaha SH, Sarhane KA, Mundinger GS, Broyles JM, Reddy SK, Azoury SC, et al. Pyoderma Gangrenosum after Breast Surgery: Diagnostic Pearls and Treatment Recommendations Based on a Systematic Literature Review. Ann Plast Surg. 2016;77(2):e39-e44. http://dx.doi.org/10.1097/ SAP.0000000000000248

15. Ehrl DC, Heidekrueger PI, Broer PN. Pyoderma gangrenosum after breast surgery: A systematic review. J Plast Reconstr Aesthetic Surg. 2018;71(7):1023-32. http://dx.doi.org/10.1016/j. bjps.2018.03.013

16. Busato WM de M, Pontes LT, Velho PENF, Magalhães RF. Pioderma gangrenoso da mama - relato de caso e aspectos relevantes para o diagnóstico precoce. Diagn Trat. 2016;21(2):65-9. 